\title{
Parents' impaired emotion recognition abilities are related to children's autistic symptoms in autism spectrum disorder
}

This article was published in the following Dove Press journal:

Neuropsychiatric Disease and Treatment

\author{
Xiao $\mathrm{Hu}^{1-3, *}$ \\ Li Yin ${ }^{1-3, *}$ \\ Mingjing Situ ${ }^{1-3}$ \\ Kuifang Guo ${ }^{1-3}$ \\ Pingyuan Yang ${ }^{1-3}$ \\ Manxue Zhang ${ }^{1-3}$ \\ Yi Huangl-3 \\ 'Department of Psychiatry, Mental \\ Health Center, West China Hospital \\ of Sichuan University, Chengdu \\ 6I004I, Sichuan, China; ${ }^{2}$ Psychiatric \\ Laboratory, State Key Laboratory \\ of Biotherapy, West China Hospital, \\ Sichuan University, Chengdu 61004I, \\ Sichuan, China; ${ }^{3}$ Brain Research \\ Center, West China Hospital of \\ Sichuan University, Chengdu 61004I, \\ Sichuan, China \\ *These authors contributed equally \\ to this work
}

Objective: We aimed to explore whether parents of children with autism spectrum disorder (ASD) had impaired emotion recognition abilities and whether this deficit was related to their children's autistic symptoms.

Methods: The autistic symptoms of 31 ASD children were assessed using the Autism Diagnostic Interview-Revised (ADI-R). Fifty parents of ASD children and 34 parents of typically developing (TD) children completed an emotion recognition task (ERT).

Results: The numbers of correct ERT responses were lower for parents of ASD children than for parents of TD children with respect to recognizing sadness, disgust, fear, and all emotions $(P=0.01,0.04,0.02$, and 0.00, respectively). Controlled for parental age, gender, and the intelligence quotients of both the parents and children, a negative correlation was found between the total number of correct ERT responses for parents of ASD children and these children's "restricted, repetitive, and stereotyped patterns of behavior" scores on the ADI-R ( $r=-0.32$; $P=0.03$ ).

Conclusion: Parents of ASD children showed impaired emotion recognition abilities compared with parents of TD children. This parental deficit in emotion recognition ability was related to the autistic symptoms of ASD children.

Keywords: autism spectrum disorder, parents, emotion recognition

\section{Introduction}

Autism spectrum disorder (ASD) is a neurodevelopmental disorder characterized by deficits in social communication and the presence of stereotyped repetitive interests and activities. ${ }^{1}$ The Autism and Developmental Disabilities Monitoring Network in USA reported that in 2014, the prevalence of ASDs had increased to 1 in $68 ; 2,3$ the corresponding prevalence in Mainland China in 2014 was $0.28 \%-6.22 \% .{ }^{4,5}$ ASD, a lifelong disorder, imposes heavy health and economic burdens on individuals, families, and societies. ${ }^{6,7}$

Functional deficits in social cognition have been found in ASD ranging from toddlers to adults, ${ }^{7,8}$ and facial emotion recognition is one of the most important parts of social-cognitive function. ${ }^{9}$ Loukusa et $\mathrm{al}^{10}$ found that ASD children had impaired emotion recognition compared with typically developing (TD) children or children with specific language impairment. Sachse et $\mathrm{al}^{11}$ found that high-functioning ASD children performed worse with respect to recognizing simple and complex facial emotions than did patients with paranoid schizophrenia or TD individuals. Certain metaanalysis studies have also reported that ASD children exhibit facial emotion recognition deficits across multiple expressions, especially when observing negative emotions,
Correspondence: Yi Huang

Department of Psychiatry, Mental Health Center, West China Hospital of Sichuan University, No. 37 Guo Xue Xiang,

Chengdu 61004I, China

Tel +862885422023

Fax +86 2885582944

Email huangyu@scu.edu.cn 
and that these deficits are independent of intelligence. ${ }^{12-14}$ Moreover, a functional magnetic resonance imaging (fMRI) study indicated that the emotion recognition deficits in ASD children could be predicted by hypoconnectivity of the superior temporal sulcus. ${ }^{15}$ In early research on ASD, the superior temporal sulcus was implicated in eye gaze processing and voice perception. The amygdala has also been linked to emotion recognition and theory of mind deficits in autism, while the fusiform gyrus has been proposed as a mechanism underlying face processing deficits in autism. ${ }^{16}$ Besides gray matter, white matter tracts of the socioemotional processing system were also found to be abnormally connected, such as the longitudinal fasciculus, splenium fibers, and the optic tract. ${ }^{17}$ Both, functional and anatomical abnormalities have been observed in social cognition brain areas in ASD.

In addition, individuals with autism and their parents and siblings all have impaired emotion recognition abilities. The study by Bölte and Poustka ${ }^{18}$ reported that there was a tendency for subjects from multiplex families with autistic loading to score lower on emotion recognition tasks (ERTs) than individuals from simplex families with autistic loading, and the extent of facial recognition deficits likely indexes an elevation in familial burden. Other studies exploring the association between facial emotion recognition deficits and functional polymorphism of the serotonin transporter (5HTTLPR) have reported that 5HTTLPR polymorphism may determine different error patterns. ${ }^{19}$ Hence, we suggest that facial emotion recognition is a candidate endophenotype for autism. Endophenotypes are heritable markers associated with a given condition and can provide insight into its etiology. ${ }^{20}$ The study of endophenotypes is particularly useful in understanding autism because they are diagnosed based on the clinical features but are of neurobiological origin and can aid in better identifying and characterizing the nature of the genetic contributions to this complex disorder.

However, there is no consensus regarding emotion recognition deficits in parents of ASD children. Palermo et $\mathrm{al}^{21}$ found that parents of ASD children performed worse on an ERT than did parents of TD children. Kadak et $\mathrm{al}^{22}$ reported that parents of ASD children had difficulty in recognizing neutral facial expressions. However, Bölte and Poustka ${ }^{18}$ found that parents of ASD children did not differ from healthy controls with respect to their ability to judge facial affect. According to the studies by Kadak et $\mathrm{al}^{22}$ and Adolphs et al, ${ }^{23}$ the positive results of these two studies may have been caused by the failure to control demographic differences (eg, education years and intelligence quotients [IQs]) and the difficulty of the ERTs. Furthermore, the study in the early time by Palermo et $\mathrm{al}^{21}$ only matched control parents by educational level and not on the basis of a standardized test of intelligence. The experiment materials used in these three studies may also contribute to the different results reported. The study by Palermo et $\mathrm{al}^{21}$ used schematic line drawings of male faces rather than real pictures, similar to that used by Kadak et $\mathrm{al}^{22}$ and Adolphs et al. ${ }^{23}$ In addition, the previous studies investigated only the relationships between symptoms of parents and their emotion recognition. Exploring the relationship between the emotion recognition abilities of parents of ASD children and these children's autistic symptoms may help us better understand the role of heredity and other variations in autism.

\section{Methods}

\section{Participants}

All the participants were consecutive from the beginning. Thirty-one ASD children ( 28 males/3 females) were recruited from the outpatient department of West China Hospital of Sichuan University (24 ASD children), a special education school (four ASD children), and a normal primary school (three ASD children) in Chengdu, China. Three experienced psychiatrists diagnosed and assessed these children using the Diagnostic and Statistical Manual of Mental Disorders, fifth edition (DSM-V) and the Autism Diagnostic InterviewRevised (ADI-R). All the psychiatrists completed training courses for the ADI-R. Interrater reliability was evaluated using the kappa coefficient (kappa=0.85).

Twenty-four TD children (21 males/3 females) were recruited from three normal primary schools via posted advertisements. Neither the ASD children nor the TD children had any comorbid medical or neurological conditions such as fragile $\mathrm{X}$ syndrome. All the ASD and TD children were assessed using the Kiddie Schedule for Affective Disorders and Schizophrenia for School-Age Children Present and Lifetime version (K-SADS-PL). Nine of the ASD children were diagnosed with attention deficit/hyperactivity disorder (ADHD). None of the TD children were diagnosed with any other psychiatric disorders. Fifty parents of ASD children (26 males/24 females) and 34 parents of TD children (14 males/20 females) were recruited. None of the parents had any psychiatric disorders. This study was approved by the ethical committee of West China Hospital and followed the guidelines set forth in the Declaration of Helsinki. All subjects signed informed consent forms and understood the precise purposes and processes of the research prior to participating in this study.

\section{Cognitive assessment}

The Chinese version of the Wechsler Intelligence Scale III was used to evaluate the intelligence of all parents and 
children. Verbal intelligence quotient (VIQ), performance intelligence quotient (PIQ), and total IQ were recorded.

The parents of ASD and TD children were presented with the ERT component of the Cambridge Neuropsychological Test Automated Battery (CANTAB) Eclipse Test (Administration Guide Manual version 5.0.0, http://www. cambridgecognition.com/), which involved a set of computerized paradigms run on a Lenovo-compatible computer with a high-resolution color monitor and a touch-sensitive screen. The ERT, developed at the University of Bristol by Professor Marcus Munafõ and Dr Ian Penton-Voak, is a task of social cognition that measures the ability to identify emotions in facial expressions and was validated in a previous study by Yip and Lee. ${ }^{24}$ It was also used in a study on emotion recognition in Chinese people with schizophrenia. ${ }^{25}$ The parents were shown a series of faces that were computer-morphed images derived from facial features of actual individuals. Each face showed a specific emotion, and the faces were displayed onscreen, one at a time, in a random order. Each face was displayed for $200 \mathrm{~ms}$ and was then immediately obscured to prevent residual processing of the image. The participant was required to select which emotion the face displayed from six options (sadness, happiness, fear, anger, disgust, and surprise). There were two blocks, each containing 90 faces (15 faces per emotion). The number of correct responses for each emotion and the total number of correct responses for the recognition of all emotions were measured.

\section{Data analyses}

The SPSS version 23.0 for Windows (IBM Corporation, Armonk, NY, USA; www.ibm.com/software/analytics/ spss/) was used for statistical analyses. Descriptive statistics were used for demographic and clinical variables. Gender, parent age at the birth of the relevant child, family history of mental disorder, and family income were analyzed using chi-squared tests; age and years of education as well as IQ, ERT, and ADI-R scores were analyzed using two-sample $t$-tests. Partial correlation analysis was conducted to relate ERT results that significantly differed between groups with ADI-R scores of ASD children, controlled for parental age, gender, and IQ. Bonferroni correction was performed to control the rate of type I errors when conducting multiple correlations. All the tests were two tailed, with a significance threshold of $P<0.05$.

\section{Results}

\section{Demographic and clinical characteristics}

There were no significant differences in age $(t=1.10 ; P=0.29)$, gender $(t=1.29 ; P=0.26)$, or family income $\left(\chi^{2}=5.57 ; P=0.14\right)$ between ASD children and TD children. A family history of mental illness was more common in ASD children than in TD children $\left(\chi^{2}=4.63 ; P=0.03\right)$, and ASD children had lower VIQ, PIQ, and total IQ scores than TD children ( $t=-5.57,-6.90$, and -6.39 , respectively; $P=0.00$ for all). The parents of ASD and TD children did not significantly differ with respect to age, gender, age when the relevant child was born, years of education, VIQ, PIQ, or total IQ $(t=1.75,0.95,2.96,0.92,0.33,0.45$, and 0.45 , respectively; $P=0.09,0.33,0.40,0.36,0.74,0.66$, and 0.66 , respectively; Tables 1 and 2).

\section{ERT scores for parents of ASD children and parents of TD children}

The number of correct ERT responses was lower for parents of ASD children than for parents of TD children with respect to recognizing sadness, disgust, fear, and all emotions $(t=-2.79,-2.10,-2.39$, and -3.23 , respectively; $P=0.01,0.04$, 0.02 , and 0.00 , respectively). However, the number of correct ERT responses did not significantly differ between these two groups with respect to recognizing happiness, anger, or surprise (Table $2 ; t=-0.63,-1.43$, and -0.71 , respectively; $P=0.54,0.15$, and 0.48 , respectively).

\section{Correlation between total number of correct ERT responses by parents of ASD children and these children's ADI-R scores}

We conducted partial correlation analysis relating ERT results that significantly differed between groups with the ADI-R scores of ASD children, controlled for parental age, gender, and the IQs of both the parents and children. A negative correlation was found between the total number of correct ERT responses for all six emotions by parents of ASD children and these children's "repetitive behaviors and stereotyped pattern" scores on the ADI-R ( $r=-0.32$; $P=0.03$ ) before Bonferroni correction (Figures 1 and 2). These parents' total number of correct ERT responses was not significantly related to the ASD children's scores on the "social and communication" subscales of the ADI-R $(r=-0.12 ; P=0.13)$.

\section{Discussion}

We found that parents of ASD children performed worse than parents of TD children with respect to recognizing sadness, fear, and disgust during the ERT. This finding suggested that parents of ASD children exhibited impaired emotion recognition abilities for negative emotions and that this impairment is associated with autism. Our result is similar to the findings reported by Kadak et al, ${ }^{22}$ Wallace et al, ${ }^{26}$ 
Table I Demographic and clinical characteristics of ASD and TD children

\begin{tabular}{|c|c|c|c|c|c|c|}
\hline & \multicolumn{2}{|c|}{$\begin{array}{l}\text { ASD children } \\
(n=31)\end{array}$} & \multicolumn{2}{|c|}{$\begin{array}{l}\text { TD children } \\
(n=24)\end{array}$} & \multirow[t]{2}{*}{$\chi^{2} / t$} & \multirow[t]{2}{*}{$P$-value } \\
\hline & $\mathbf{n}$ & $\%$ & $\mathbf{n}$ & $\%$ & & \\
\hline \multicolumn{7}{|l|}{ Gender } \\
\hline Male & 28 & 90.32 & 21 & 87.50 & 1.29 & 0.26 \\
\hline Female & 3 & 9.68 & 3 & 12.50 & & \\
\hline \multicolumn{7}{|l|}{ Family income ( $¥ \mathrm{CNY}$ ) } \\
\hline$<3,000$ & 12 & 24.00 & 5 & $14.7 \mid$ & 5.57 & 0.14 \\
\hline $3-5,000$ & 16 & 32.00 & 9 & 26.47 & & \\
\hline $5-8,000$ & 11 & 22.00 & 13 & 38.23 & & \\
\hline$>8,000$ & 11 & 22.00 & 7 & 20.59 & & \\
\hline \multirow[t]{2}{*}{ Family history } & 8 & 25.82 & 1 & 4.17 & 4.63 & $0.03^{*}$ \\
\hline & Mean & SD & Mean & SD & $\chi^{2} / t$ & $P$-value \\
\hline Age (years) & 8.57 & 2.29 & 7.43 & 2.19 & 1.10 & 0.29 \\
\hline \multicolumn{7}{|l|}{ IQ } \\
\hline VIQ & 69.70 & 19.95 & 97.50 & 15.78 & -5.57 & $0.00 * *$ \\
\hline PIQ & 64.53 & 17.04 & 93.16 & 11.94 & -6.90 & $0.00 * *$ \\
\hline Total & 66.56 & 18.49 & 95.50 & 13.69 & -6.39 & $0.00 * *$ \\
\hline \multicolumn{7}{|l|}{ ADI-R } \\
\hline Communication & 23.12 & 8.71 & NA & NA & & \\
\hline Reciprocal social interaction & 18.88 & 6.60 & NA & NA & & \\
\hline Repetitive behaviors and stereotyped patterns & 3.36 & 2.84 & NA & NA & & \\
\hline Total score & 48.24 & 16.05 & NA & NA & & \\
\hline
\end{tabular}

Notes: $* P<0.05 ; * * P<0.01$.

Abbreviations: ADI-R, Autism Diagnostic Interview-Revised; ASD, autism spectrum disorder; CNY, Chinese Yuan; IQ, intelligence quotient; NA, not applicable; PIQ, performance intelligence quotient; TD, typically developing; VIQ, verbal intelligence quotient.

and Palermo et $\mathrm{al}^{21}$ that the fathers of autistic children performed more poorly on tasks of emotion recognition than either these children's mothers or the control subjects. However, Bölte and Poustka ${ }^{18}$ found that ASD parents did not perform differently from healthy controls on an emotion recognition test. As the studies by Kadak et $\mathrm{al}^{22}$ and Adolphs et $\mathrm{a}^{23}$ failed to control for demographic differences (ie, education years and IQs) and used different statistical methods, they obtained different outcomes.

We further found that the total number of correct ERT responses provided by parents of ASD children was negatively related to these children's "restricted, repetitive, and stereotyped patterns of behavior" scores on the ADI-R. This result suggested that this important social cognition deficit of the parents of ASD children was related to these children's autistic symptom severity, particularly with respect to repetitive and stereotyped symptoms. To our knowledge, this article provides the first report of an association between a social cognition deficit in the parents of ASD children and the severity of those children's autistic symptoms, although other social behaviors or cognition phenotypes in parents have been found to be related to ASD children's autistic symptoms. ${ }^{27,28}$ It has been reported that the social skills and communication ability of ASD children's mothers were related to the severity of ASD children's autistic symptoms. ${ }^{27}$ The mitigation of autistic symptoms in ASD children has been associated with an increased empathy in their parents. ${ }^{28}$

Several studies on social cognition in mental disorders, such as schizophrenia, have indicated that impaired emorecognition ability of individuals is related to symptom severity. ${ }^{29-32}$ Deficits in emotion recognition that extend beyond the recognition of facial expressions have been associated with reciprocal social interaction symptoms in autism. ${ }^{33}$ In our study, we found an between social cognition deficit in ASD parents and ASD children's autistic symptom severity.

Our study found that the "repetitive and stereotyped symptoms" of children were correlated with parents' ability in emotion recognition, but the "social and communication" subscale was not correlated, which may seem surprising. 
Table 2 Demographic characteristics and ERT scores of ASC's and TDC's parents

\begin{tabular}{|c|c|c|c|c|c|c|}
\hline & \multicolumn{2}{|c|}{$\begin{array}{l}\text { Parents of ASC } \\
(n=50)\end{array}$} & \multicolumn{2}{|c|}{$\begin{array}{l}\text { Parents of TDC } \\
(n=34)\end{array}$} & \multirow[t]{2}{*}{$\chi^{2 / t}$} & \multirow[t]{2}{*}{$P$-value } \\
\hline & $\mathbf{n}$ & $\%$ & $\mathbf{n}$ & $\%$ & & \\
\hline \multicolumn{7}{|l|}{ Gender } \\
\hline Male & 26 & 52.00 & 14 & 41.18 & 0.95 & 0.33 \\
\hline Female & 24 & 48.00 & 20 & 58.82 & & \\
\hline \multicolumn{7}{|c|}{ Age of giving birth (years) } \\
\hline$<25$ & 11 & 22.00 & 7 & 20.58 & 2.96 & 0.40 \\
\hline $25-30$ & 11 & 22.00 & 13 & 38.24 & & \\
\hline $30-35$ & 16 & 32.00 & 9 & 26.47 & & \\
\hline \multirow[t]{2}{*}{$>35$} & 12 & 24.00 & 5 & $|4.7|$ & & \\
\hline & Mean & SD & Mean & SD & $\chi^{2} / t$ & $P$-value \\
\hline Age (years) & 36.12 & 5.46 & 34.12 & 4.67 & 1.75 & 0.09 \\
\hline Education years & 12.62 & 3.80 & 11.97 & 2.67 & 0.92 & 0.36 \\
\hline \multicolumn{7}{|l|}{ IQ } \\
\hline VIQ & 104.84 & 13.74 & 98.7I & 12.73 & 0.33 & 0.74 \\
\hline PIQ & 101.36 & $17.4 \mid$ & 100.00 & 13.08 & 0.45 & 0.66 \\
\hline Total & 103.84 & 14.07 & 99.44 & 12.73 & 0.45 & 0.66 \\
\hline \multicolumn{7}{|c|}{ Correct number of ERT } \\
\hline Happy & 22.72 & 4.07 & 23.26 & 3.45 & -0.63 & 0.54 \\
\hline Anger & 10.96 & 4.50 & 12.35 & 4.16 & -1.43 & 0.15 \\
\hline Sad & 15.82 & 6.18 & 18.13 & 4.35 & -2.79 & $0.01 *$ \\
\hline Disgust & 10.70 & 5.82 & 13.23 & 4.81 & -2.10 & $0.04 *$ \\
\hline Fear & 7.90 & 4.13 & 10.59 & 5.60 & -2.39 & $0.02 *$ \\
\hline Surprise & 19.26 & 4.43 & 19.94 & 4.21 & $-0.7 I$ & 0.48 \\
\hline Total & 88.10 & $13.2 \mid$ & 98.18 & 16.08 & -3.23 & $0.00 * *$ \\
\hline
\end{tabular}

Notes: $* P<0.05 ; * * P<0.01$.

Abbreviations: ASC, autism spectrum disorder children; ERT, emotion recognition task; IQ, intelligence quotient; PIQ, performance intelligence quotient; TDC, typically developing children; VIQ, verbal intelligence quotient.

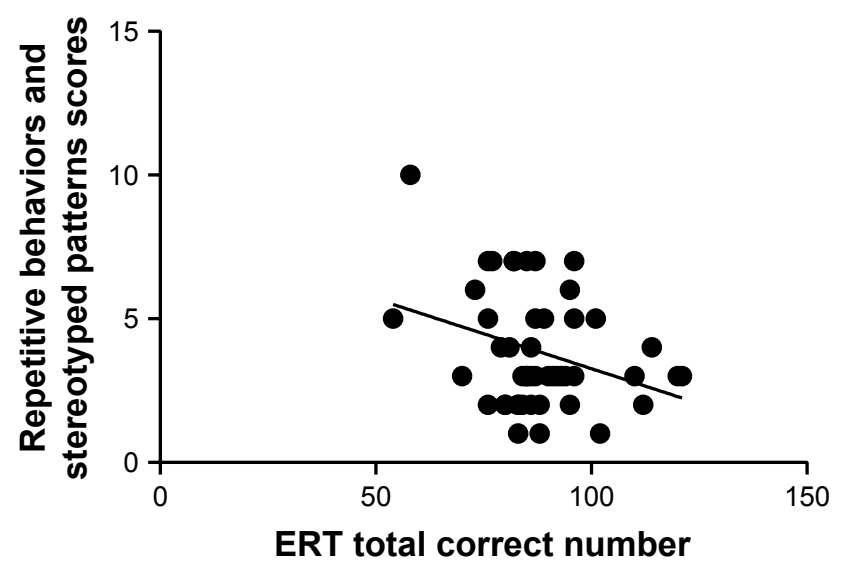

Figure I Negative correlation was found between the total number of correct ERT responses for recognizing all six types of emotions by parents of ASD children and these children's "repetitive behaviors and stereotyped pattern" scores on the ADI-R ( $r=-0.32$; $P=0.03$ ), controlled for parental age, gender, and IQ of both the parents and children.

Abbreviations: ADI-R, Autism Diagnostic Interview-Revised; ASD, autism spectrum disorder; ERT, emotion recognition task; IQ, intelligence quotient.
Studies on sibling correlations have consistently shown that repetitive behaviors are correlated among siblings, while impairments in social reciprocity are not. ${ }^{34}$ In addition, it is usually repetitive behaviors for which significant linkage results are reported in sub-type linkage analyses of ASD. ${ }^{35}$ A phenotypic homogeneity analysis by Hus et $\mathrm{al}^{36}$ reported that only restricted and repetitive behaviors associated with insistence on sameness were independent of age, IQ, and autism severity. Above all, we suggest that the repetitive and stereotyped symptoms were more highly and stably hereditary than social and communication symptoms.

These findings suggested that in the future, the social cognition deficits of ASD parents may be used to predict symptoms severity for their children, even when these children are at extremely early stages and are not exhibiting many symptoms, and to screen high-risk subjects. Our results 


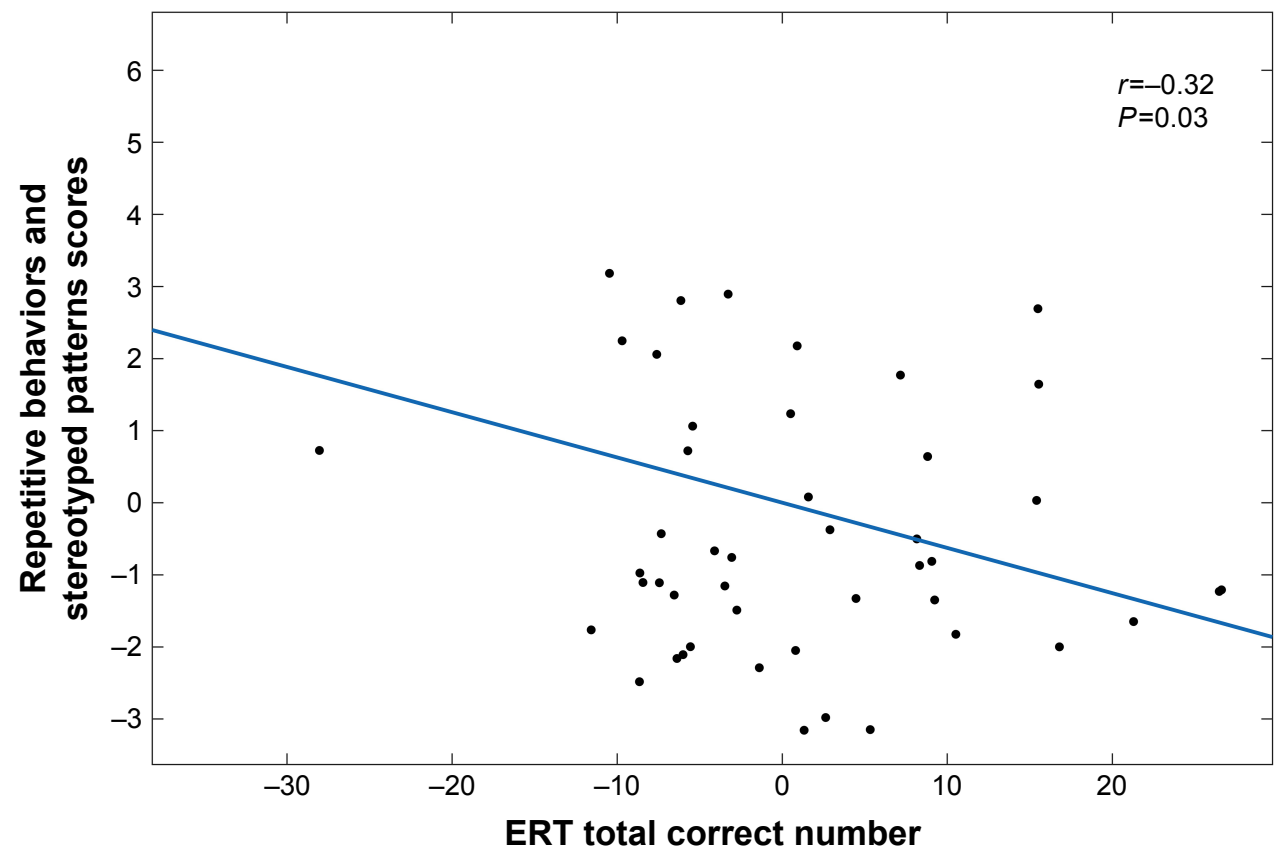

Figure 2 Partial correlation plot showed the negative correlation between the total number of correct ERT responses for recognizing all six types of emotions by parents of ASD children and these children's "repetitive behaviors and stereotyped pattern" scores on the ADI-R ( $r=-0.32$; $P=0.03)$, controlled for parental age, gender, and IQ of both the parents and children.

Abbreviations: ADI-R, Autism Diagnostic Interview-Revised; ASD, autism spectrum disorder; ERT, emotion recognition task; IQ, intelligence quotient.

may also help facilitate early diagnosis of ASD in toddlers, which is a challenging population for ASD diagnosis.

In terms of the underlying mechanism explaining our results, evidence from neuroimages has shown that certain brain regions (such as the amygdala) are responsible for both emotion cognition and repetitive, stereotyped symptoms in autism and their relatives. ${ }^{26,37}$ Animal studies have revealed different neuronal subsets in the amygdala, namely, the GABAergic and glutamatergic subpopulations. The GABAergic subpopulation promotes aggression and two other social behaviors, whereas neighboring glutamatergic neurons promote repetitive self-grooming. ${ }^{38}$ These results suggest that social behaviors and repetitive and social behaviors are controlled in an antagonistic manner by inhibitory and excitatory amygdala subpopulations, respectively. ${ }^{39}$

There is an explanation for the uncorrelated relationship between parents' emotion recognition and children's social impairment symptoms: emotion recognition may be an intermediate phenotype rather than a specific feature of autistic symptoms. As Cook et $\mathrm{al}^{40}$ reported, emotion recognition is related to alexithymia rather than autistic traits. Based on the report by Bird and $\mathrm{Cook}^{41}$ on ASD individuals with alexithymia representing a subgroup of autism, we can draw the same conclusion as Szatmari et $\mathrm{al}^{42}$ that the alexithymia trait represented by emotion recognition impairment makes up the broader autism phenotype of parents of ASD children. What is more, children's autistic symptoms can also be aggravated in family life. Other studies have explored how the emotional reaction of parents affects the behavior and emotional competence of children in normal populations. ${ }^{43,44}$

Unfortunately, in this study, we did not obtain sufficient information regarding the ERT performance of ASD children because only a few of these children completed the ERT assessment. To find more substantial evidence of association between emotion recognition and autistic symptoms in children and emotion, we need to obtain additional information about ASD children's ERT performance in the future.

\section{Conclusion}

Our study found impaired emotion recognition abilities in parents of ASD children, and this impairment was associated with these children's autistic symptoms. Our research suggested that the emotion recognition ability deficits of ASD parents may be an important cognition phenotype in ASD families and could be a promising biomarker for predicting symptom severity.

\section{Ethics approval and consent to participate}

Before taking part in the study, all participants were informed about the study and provided written informed consent, 
which was approved by the Medical Research Ethics Committee of West China Hospital, Sichuan University.

\section{Informed consent}

Consent for the publication of clinical data and information was obtained.

\section{Acknowledgments}

This study was supported by the National Natural Science Foundation of China (No 81371495), the Public Project Foundation of the National Health and Family Planning Commission of the PRC (No 201302002), Key Research Project from Ministry of Science and Technology China (No 2016YFC1306104), and Key Project from Sichuan Provincial Science and Technology Department (No 2017SZ0135). This research received no specific grant from any funding agency or from the commercial or not-for-profit sector. The authors gratefully acknowledge the contributions of Ms Chen Meiyu and Ms Liao Xingjun.

\section{Author contributions}

All authors contributed to data analysis, drafting and revising the article, gave final approval of the version to be published, and agree to be accountable for all aspects of the work.

\section{Disclosure}

The authors report no conflicts of interest in this work.

\section{References}

1. Tchaconas A, Adesman A. Autism spectrum disorders: a pediatric overview and update. Curr Opin Pediatr. 2013;25(1):130-144.

2. Developmental Disabilities Monitoring Network Surveillance Year 2010 Principal InvestigatorsCenters for Disease Control and Prevention (CDC). Prevalence of autism spectrum disorder among children aged 8 years autism and developmental disabilities monitoring network, 11 sites, United States, 2010. MMWR Surveill Summ. 2014;63(2):1-21.

3. Autism and Developmental Disabilities Monitoring Network Surveillance Year 2008 Principal InvestigatorsCenters for Disease Control and Prevention. Prevalence of autism spectrum disorders - Autism and Developmental Disabilities Monitoring Network, 14 sites, United States, 2008. MMWR Surveill Summ. 2012;61(3):1-19.

4. Sun X, Allison C, Auyeung B, et al. Comparison between a Mandarin Chinese version of the Childhood Autism Spectrum Test and the Clancy Autism Behaviour Scale in mainland China. Res Dev Disabil. 2014; 35(7):1599-1608.

5. Jiang L, Li G, Hao L, Guo R, Yang C, du Y. Epidemiological investigation on autism spectrum disorders among preschool children in Shanghai. Zhonghua Liu Xing Bing Xue Za Zhi. 2015;36(12):1365-1368.

6. Samadi SA, Mcconkey R. The impact on Iranian mothers and fathers who have children with an autism spectrum disorder. J Intellect Disabil Res. 2014;58(3):243-254.

7. Blanchard LT, Gurka MJ, Blackman JA, Emotional BJA. Emotional, developmental, and behavioral health of American children and their families: a report from the 2003 National Survey of Children's Health. Pediatrics. 2006;117(6):e1202-e1212.
8. Kimhi Y, Shoam-Kugelmas D, Agam Ben-Artzi G, Ben-Moshe I, Bauminger-Zviely $\mathrm{N}$. Theory of mind and executive function in preschoolers with typical development versus intellectually able preschoolers with autism spectrum disorder. J Autism Dev Disord. 2014;44(9):2341-2354.

9. Brunelle F, Saitovitch A, Boddaert N, et al. Human interaction, social cognition, and the superior temporal sulcus. Bull Acad Natl Med. 2013; 197(4-5):817-828.

10. Loukusa S, Mäkinen L, Kuusikko-Gauffin S, Ebeling H, Moilanen I. Theory of mind and emotion recognition skills in children with specific language impairment, autism spectrum disorder and typical development: group differences and connection to knowledge of grammatical morphology, word-finding abilities and verbal working memory. Int $J$ Lang Commun Disord. 2014;49(4):498-507.

11. Sachse M, Schlitt S, Hainz D, et al. Facial emotion recognition in paranoid schizophrenia and autism spectrum disorder. Schizophr Res. 2014;159(2-3):509-514.

12. Uljarevic M, Hamilton A. Recognition of emotions in autism: a formal meta-analysis. J Autism Dev Disord. 2013;43(7):1517-1526.

13. Lozier LM, Vanmeter JW, Marsh AA. Impairments in facial affect recognition associated with autism spectrum disorders: a meta-analysis. Dev Psychopathol. 2014;26(4 Pt 1):933-945.

14. Williams BT, Gray KM. The relationship between emotion recognition ability and social skills in young children with autism. Autism. 2013; 17(6):762-768.

15. Alaerts K, Woolley DG, SteyaertJ, di Martino A, Swinnen SP, WenderothN. Underconnectivity of the superior temporal sulcus predicts emotion recognition deficits in autism. Soc Cogn Affect Neurosci. 2014;9(10): 1589-1600.

16. Pelphrey K, Adolphs R, Morris JP. Neuroanatomical substrates of social cognition dysfunction in autism. Ment Retard Dev Disabil Res Rev. 2004;10(4):259-271.

17. Ameis SH, Catani M. Altered white matter connectivity as a neural substrate for social impairment in Autism Spectrum Disorder. Cortex. 2015;62:158-181.

18. Bölte S, Poustka F. The recognition of facial affect in autistic and schizophrenic subjects and their first-degree relatives. Psychol Med. 2003;33(5):907-915.

19. Neves MC, Tremeau F, Nicolato R, Lauar H, Romano-Silva MA, Correa H. Facial emotion recognition deficits in relatives of children with autism are not associated with 5HTTLPR. Rev Bras Psiquiatr. 2011;33(3):261-267.

20. Gottesman II, Gould TD. The endophenotype concept in psychiatry: etymology and strategic intentions. Am J Psychiatry. 2003;160(4): 636-645.

21. Palermo MT, Pasqualetti P, Barbati G, Intelligente F, Rossini PM. Recognition of schematic facial displays of emotion in parents of children with autism. Autism. 2006;10(4):353-364.

22. Kadak MT, Demirel OF, Yavuz M, Demir T. Recognition of emotional facial expressions and broad autism phenotype in parents of children diagnosed with autistic spectrum disorder. Compr Psychiatry. 2014; 55(5):1146-1151.

23. Adolphs R, Spezio ML, Parlier M, Piven J. Distinct face-processing strategies in parents of autistic children. Curr Biol. 2008;18(14):1090-1093.

24. Yip JT, Lee TM. Cultural difference in facial emotion recognition. Brain and Cognition.2003.51:169-170.

25. Chan CC, Wong R, Wang K, Lee TM. Emotion recognition in Chinese people with schizophrenia. Psychiatry Res. 2008;157(1-3):67-76.

26. Wallace S, Sebastian C, Pellicano E, Parr J, Bailey A. Face processing abilities in relatives of individuals with ASD. Autism Res. 2010;3(6): $345-349$.

27. Hasegawa C, Kikuchi M, Yoshimura Y, et al. Broader autism phenotype in mothers predicts social responsiveness in young children with autism spectrum disorders. Psychiatry Clin Neurosci. 2015;69(3):136-144.

28. Hasegawa C, Kikuchi M, Yoshimura Y, et al. Changes in autistic trait indicators in parents and their children with ASD: A preliminary longitudinal study. Psychiatry Res. 2015;228(3):956-957. 
29. Lochner C, Simmons C, Kidd M, et al. Differential effects of escitalopram challenge on disgust processing in obsessive-compulsive disorder. Behav Brain Res. 2012;226(1):274-280.

30. Corcoran KM, Woody SR, Tolin DF. Recognition of facial expressions in obsessive-compulsive disorder. J Anxiety Disord. 2008;22(1): 56-66.

31. Grisham JR, Henry JD, Williams AD, Bailey PE. Socioemotional deficits associated with obsessive-compulsive symptomatology. Psychiatry Res. 2010;175(3):256-259.

32. Larøi F, Fonteneau B, Mourad H, Raballo A. Basic emotion recognition and psychopathology in schizophrenia. J Nerv Ment Dis. 2010; 198(1):79-81.

33. Boraston Z, Blakemore SJ, Chilvers R, Skuse D. Impaired sadness recognition is linked to social interaction deficit in autism. Neuropsychologia. 2007;45(7):1501-1510.

34. Szatmari P, Georgiades S, Bryson S, et al. Investigating the structure of the restricted, repetitive behaviours and interests domain of autism. $J$ Child Psychol Psychiatry. 2006;47(6):582-590.

35. Shao Y, Cuccaro ML, Hauser ER, et al. Fine mapping of autistic disorder to chromosome 15q11-q13 by use of phenotypic subtypes. Am J Hum Genet. 2003;72(3):539-548.

36. Hus V, Pickles A, Cook EH, Risi S, Lord C. Using the autism diagnostic interview - revised to increase phenotypic homogeneity in genetic studies of autism. Biol Psychiatry. 2007;61(4):438-448.
37. Ashwin C, Chapman E, Colle L, Baron-Cohen S. Impaired recognition of negative basic emotions in autism: a test of the amygdala theory. Soc Neurosci. 2006;1(3-4):349-363.

38. Hong W, Kim DW, Anderson DJ. Antagonistic control of social versus repetitive self-grooming behaviors by separable amygdala neuronal subsets. Cell. 2014;158(6):1348-1361.

39. Breece E, Paciotti B, Nordahl CW, et al. Myeloid dendritic cells frequencies are increased in children with autism spectrum disorder and associated with amygdala volume and repetitive behaviors. Brain Behav Immun. 2013;31:69-75.

40. Cook R, Brewer R, Shah P, Bird G, Alexithymia BG. Autism not. Alexithymia, not autism, predicts poor recognition of emotional facial expressions. Psychol Sci. 2013;24(5):723-732.

41. Bird G, Cook R. Mixed emotions: the contribution of alexithymia to the emotional symptoms of autism. Transl Psychiatry. 2013;3:e285.

42. Szatmari P, Georgiades S, Duku E, Zwaigenbaum L, Goldberg J, Bennett T. Alexithymia in parents of children with autism spectrum disorder. J Autism Dev Disord. 2008;38(10):1859-1865.

43. Root AK, Rubin KH. Gender and parents' reactions to children's emotion during the preschool years. New Dir Child Adolesc Dev. 2010; 2010(128):51-64.

44. Castro VL, Halberstadt AG, Lozada FT, Craig AB. Beliefs parents' emotion-related. behaviors, and skills predict children's recognition of emotion. Infant Child Dev. 2015;24(1):1-22.
Neuropsychiatric Disease and Treatment

\section{Publish your work in this journal}

Neuropsychiatric Disease and Treatment is an international, peerreviewed journal of clinical therapeutics and pharmacology focusing on concise rapid reporting of clinical or pre-clinical studies on a range of neuropsychiatric and neurological disorders. This journal is indexed on PubMed Central, the 'PsycINFO' database and CAS,

\section{Dovepress}

and is the official journal of The International Neuropsychiatric Association (INA). The manuscript management system is completely online and includes a very quick and fair peer-review system, which is all easy to use. Visit http://www.dovepress.com/testimonials.php to read real quotes from published authors. 